\title{
Devil in the Details: Effects of Depression on the Prosocial Response Depend on Timing and Similarity
}

\author{
Alicia Hofelich Mohr ${ }^{1,2} \cdot$ Ethan Kross ${ }^{1}$. \\ Stephanie D. Preston ${ }^{1}$
}

Received: 30 November 2015 / Revised: 7 March 2016/Accepted: 28 March 2016 /

Published online: 18 April 2016

(C) Springer International Publishing 2016

\begin{abstract}
Most accounts of empathy acknowledge the importance of shared experience, but debate continues regarding the precise mechanism and necessity of similarity for an empathic response. The perception-action model posits that empathy and understanding are processed through observers' own internal representations for states and situations, which increase in complexity with experience and produce more accurate, prosocial responses when observers and targets experience events similarly. We tested this model by measuring the degree to which past and current depression in observers influenced the perception of sad, distressed targets and the empathic, prosocial response to them. Participants with either prior or current depression perceived a greater number of types of negative affect in distressed targets. Past depression was also associated with an increased prosocial response to distressed patients if observers viewed them as similar. However, participants with current depression symptoms felt less empathy and gave less help to distressed targets. These results highlight the importance of distinguishing between past and current shared experience, with important consequences for clinical and social interactions.
\end{abstract}

Keywords Empathy $\cdot$ Altruism $\cdot$ Depression $\cdot$ Prosocial $\cdot$ Similarity $\cdot$ Perception-action

\section{Introduction}

Distress displays are difficult to ignore. Seeing another person cry, tear up, or tremble with emotion powerfully signals their distress. While such displays often elicit feelings

Stephanie D. Preston

prestos@umich.edu

1 Department of Psychology, University of Michigan, 530 Church Street, Ann Arbor, MI 48109, USA

2 Present address: College of Liberal Arts LATIS, University of Minnesota, Minneapolis, MN, USA 
of empathy in an observer, they do not always. In some cases, such displays of distress can even elicit negative responses including personal distress (Batson et al. 1987), anger and horror (Preston et al. 2013), or even aggression (Zahn-Waxler et al. 1992). A variety of factors are known to influence these diverging responses, such as the nature of the situation (e.g., Eisenberg and Fabes 1990), the personality of the observer (Davis 1983; Eisenberg and Fabes 1990; Eisenberg et al. 1998), and the degree to which the observer takes the target's perspective (Lamm et al. 2007).

It is also largely recognized that having a similar past experience to that of the person in need (the "target") is an important component of the empathic response (e.g., Barnett 1984; Barnett et al. 1986; Batson et al. 1996; Preston and de Waal 2002), but the true necessity for similar past experience, and the way in which it influences the response, is still debated. For example, shared experience does not always enhance the observer's ability to accurately understand the target's state (Hodges et al. 2010) and perceived similarity does not always predict helping when other factors like target vulnerability are more salient (Batson et al. 2005). Moreover, observers with similar past experiences do not feel equally empathic for all targets - their responses are modulated by the way targets display their need through expressed emotion (Preston et al. 2013). Thus, the role of similar experience on the empathic response is complex, leading to disagreement about its necessity (e.g., Preston and Hofelich 2012b; Eisenberg and Sulik 2012). Here we aim to illuminate the role that similar past experience plays in empathic responding, guided by the perception-action model of empathy (PAM; Preston and de Waal 2002).

\section{Perception-Action Model}

The perception-action model of empathy (PAM; Preston and de Waal 2002) assumes that empathizing with another's emotion involves activating the same mental representations that give rise to observers' first-hand experience of emotion. These shared representations include conceptual knowledge, motor and sensory information, episodic memories, emotions, and physiological responses associated with the relevant experience. When another's situation is appraised as similar to one's own, these multi-modal representations should encode richer, more accurate representations of the target's state and situation, producing greater understanding, empathy, and tailored care (Preston and de Waal 2002; Preston and Hofelich 2012a, 2012b).

If this is the case, then why does similarity not always influence the empathic response or does it seem less influential than other factors? According to the PAM, inconsistent relationships between similar experience and empathy arise because most research only examines superficial dimensions of similarity, such as gender (Feshbach and Roe 1968), personal interests (Krebs 1975; Rosekrans 1967), university affiliation (Batson et al. 1997) or shared life events like giving birth or going through a divorce (Hodges 2005; Hodges et al. 2010). In reality, our ability to relate to others depends critically upon the content of our mental representations, which may not vary as much with what we experience as with how we experience it (Preston and de Waal 2002; Preston and Hofelich 2012a). As such, superficial dimensions of similarity like group membership can fail to enhance the empathic response when the observer and target do not personally experience, appraise, characterize, or respond to the event the same way. For example, a childless young woman who ends a short marriage amicably may have a very different experience of divorce - and therefore different mental representations 
and feelings associated with the event - than a stay-at-home mother abandoned by her husband of 15 years after his extramarital affair. Thus, rather than comparing empathic responses on the basis of shared nominal experiences, the PAM suggests that it is more predictive to compare empathic responses on the basis of shared psychological experiences (for similar argument in another domain, see Mischel and Shoda 1995).

\section{Depression as a Case Study of Experience with Sadness}

One example of a personal, psychological, and affective experience is the intense sadness associated with clinical depression. Depression is one of the most common mental illnesses (Substance Abuse and Mental Health Services Administration 2006), especially for females (Blazer et al. 1994). Intense negative mood is a consistent, core aspect of depression, rendering it a good candidate for ensuring that people who have had depression have experienced tremendous sadness or distress. At the same time, depression can manifest differently across individuals - such as with undereating or overeating, not sleeping or sleeping too much. This means that some sufferers of depression can better relate to targets that experience sadness or distress in a way that they can relate to than to those who experience a dissimilar form. According to the PAM, the extensive experience with negative affect will endow individuals who have experienced depression with a richer representation of negative affect that, in turn, should also render them more understanding of and helpful toward targets experiencing similar distress.

This hypothesis has not been tested directly yet, as much of the literature on depression and empathy has focused on examining the development of empathy in infants of depressed mothers (Field 1995; Jones et al. 2000) or the impact of depression on trait empathy (Davis 1980; Gawronski and Privette 1997; O’Connor et al. 2002, 2007). These studies have yielded mixed results regarding whether depression decreases (Jones et al. 2000), increases (O’Connor et al. 2007), or does not influence (O’Connor et al. 2002) empathy.

A prior depression experience is expected to produce more elaborated mental representations of sad affect that should, in turn, produce: 1) more nuanced and differentiated perceptions of others' sad affect and, 2) greater prosocial responses to similar distraught patients. To test these hypotheses, participants with and without a prior depression diagnosis watched 14 videos of hospital patients with serious chronic or terminal illness being interviewed about quality of life issues. A subset of these highly distressed target patients were previously classified as distraught. Observers uniformly recognized the high level of distress and need in these targets, but only some felt more empathy for them and offered more help to them than to happy, resilient patients who appeared to need less help (see Hauser et al. 2014; Preston et al. 2013). After the current participants viewed all patient videos, they classified target patients by their affective display and rated how much they wanted to help each one with support, assistance, and real money. Participants with a prior depression experience were expected to create more categories from patients exhibiting negative affect and to report greater empathy and willingness to help them.

Additional testing and analyses were also performed to determine if results were differently influenced by current - as opposed to prior-depression, because current depression is known to render people more self-oriented, which could counteract the benefits of having had a similar experience with sadness. Thus, in addition to measuring group differences from having or not having a prior depression experience, all 
analyses also examined the relationship between continuous current depression severity across the whole population. Finally, to discern whether any effects of depression emanated from altered physiological reactivity (Bylsma et al. 2008) or an attentional bias to the distraught patients (Fritzsche et al. 2010; Joormann and Gotlib 2007; Paelecke-Habermann et al. 2005; but see also Cheng et al. 2015 for null effects) we also compared effects of depression status on psychophysiological responses during the videos and on a separate Attentional Capture task (McKenna and Sharma 1995).

\section{Methods}

\section{Participants}

Sixty-three females $\left(M_{\text {age }}=18.8, \mathrm{SD}=0.94\right.$; range $\left.=18-21\right)$ participated for course credit, were tested individually, and gave full informed consent. Female participants were tested because depression is more common in females than males (Blazer et al. 1994). Participants were prescreened with the question "Have you ever been diagnosed with depression?" and then divided into those with a prior diagnosis of depression (prior depression experience group; 31 participants; 12 currently on medication for depression) and without a prior diagnosis (without depression experience group; 32 participants; one taking Zoloft without a diagnosis; results remain without this participant).

The current experience of depression (degree of symptoms currently experienced by each individual) was measured and analyzed with the BDI-II, which all participants completed at the end of the study (hereafter BDI; Beck et al. 1988). Indicating the validity of this approach, an independent welch t-test confirmed higher BDI scores in the prior depression experience group $(M=15.12 ; S D=11.36$, range 1-43) compared to those without depression experience $(M=7.10 ; S D=5.9$, range $0-26), t(41.76)=-3.35$ $p=.002, \mathrm{~d}=-0.88$. Moreover, BDI scores were higher for the nine individuals who reported being currently depressed $(M=28.11 ; S D=10.93$; range: $12-43)$ compared to the 22 who reported not being currently depressed $(M=9.28 ; S D=5.34$; range: $1-21)$, $t(9.75)=-4.92, p<.001, \mathrm{~d}=-2.19$. Conversely, BDI scores were similar between participants with a prior depression experience who reported not being currently depressed and participants without depression experience, $t(56.63)=-1.23, p=.23$, $\mathrm{d}=-0.26$. The average time since diagnosis in the prior depression group was 3.2 years (range $0-10$ ); time since remission ranged from 2 months to 6 years (mean $=1.2$ years), and participants said they could remember the experience $(M=5.43 / 7 ; S D=1.50)$.

\section{Overview}

Participants were seated at a Dell desktop PC and were attached to psychophysiological electrodes to record heart rate, skin conductance, and respiration. They first completed an Attentional Capture task (McKenna and Sharma 1995), and then watched the 14 patient interviews (Preston et al. 2013) in randomized order. After each video, participants rated the emotion of the patient and their own emotion while watching the video and reported how much emotional support, practical support, and donated money they would give to each. After watching all videos, participants also categorized photo cards of the patients into groups based on patients' communication and affective style. Afterwards, participants 
completed the BDI and answered questions about their experience with depression. Details on each protocol are provided below, emphasizing key measures of interest first, followed by ancillary measures that were only used to interpret results.

\section{Patient Videos}

The 14 videos contained interviews with male and female patients with a variety of serious chronic or terminal conditions that embodied a wide range of ages and emotional responses to illness, which we have used previously to study the empathic response (see Hauser et al. 2014; Preston et al. 2013). Videos ranged from $37 \mathrm{~s}$ to $2.88 \mathrm{~min}$ in length and showed only the patient, facing the camera, seated or lying back in their hospital room, from the waist up. Original interviews were edited to include the same four questions and answers from each patient, in the same order, with a brief fade between each: 1) What has been the impact of your illness on your quality of life?; 2) What are your health-related worries?; 3) What in your life are you the most proud of?; and 4) What has been the hardest thing for you to cope with related to your illness? The specific illness was not mentioned and subjects were unaware of patients' prognoses. As in previous studies (Preston et al. 2013), the 14 patients were clustered by their rated affect into fewer "types" before analysis using hierarchical clustering (Ward 1963) and an iterative relocation clustering method to finalize membership (k-means).

\section{Patient Rating and Prosocial Data}

After each video, participants recorded responses to the patient with a seven-point Likert scale from 1 (very little) to 7 (extremely). They rated how similar they felt to the patient based on the patient's situation (life similarity) and the affect they displayed (emotional similarity), as well as how sick they thought the patient was and how much they liked the patient. Participants also indicated how much practical support (e.g. getting prescriptions, changing sheets, watering plants, grocery shopping) and emotional support (e.g. talking to them, giving advice, soothing, spending time with them) they thought the patient needed and how much they were willing to give. Participants then rated 29 emotional adjectives describing the emotions they observed in the patient (other) and felt themselves while watching (self), in counterbalanced order across participants. Emotion adjectives for self and other were taken from prior work (Batson et al. 1997) but we added engaging, likeable, and entertaining to better sample positive emotions expressed by these patients. As in prior studies (Preston et al. 2013), adjectives were first condensed into other and self emotion factors using principle components analysis (PCA) with Varimax rotation. At the end of each video response period participants received five tokens, which they knew would be converted to cash at the end of the study at an unknown exchange rate, and reported how many (if any) they would donate to that patient, knowing that any undonated funds would be theirs to take home at the end.

\section{Card Sorting Task}

The card-sorting task was used to determine whether participants with a prior depression experience would sort sad, distressed patients into more groups compared to those without this experience. After watching all of the videos, participants were given 14 
cards, each of which had a picture of one of the patients, and sorted them into groups based on patient affect and communication style. They were told that we were interested in the similarities and differences that people perceive across the patients, and were instructed to place similar patients into the same group, with no restrictions on how few or many groups could be created.

\section{Depression Questionnaires}

After the experiment, participants answered experimenter questions about their history of depression (when they were diagnosed, if they were currently experiencing depression, and if not, for how long they had been in remission, and how easily they could remember what it was like to be depressed) and completed the BDI.

\section{Ancillary Measures}

Physiological Data Psychophysiological responses were collected to determine whether any behavioral effects of depression on empathy and altruism were explained by differences in autonomic arousal to the videos. Averaged responses during videos were calculated as changes from a 30s baseline measurement before the start of each video. Responses were standardized across videos for each participant to focus measurement on differential responding to distraught patients compared to other types. Mean heart rate (HR) levels were acquired with lead II EKG, with one electrode attached inferior to the costal margin and the other anterior to the sternocleidomastoid muscle. Galvanic skin response (GSR) was measured using two electrodes attached to the hypothenar area on the palm of the non-dominant hand. Because GSR rises and falls with sympathetic nervous system activity, both the mean GSR value and the number of discrete "rises" in the response (peak counts) were calculated. Mean respiration rates were calculated from the summed signal across two belts, one attached around the participant's torso and another around the upper chest. Data were sampled at a rate of $500 \mathrm{~Hz}$ using a Biopac MP150 system (Biopac Systems, Santa Barbara, California) and were recorded and preprocessed with AcqKnowledge III software for Mac (Biopac Systems). All waveforms were visually inspected for artifacts before analysis.

Attentional Capture Task In three different blocks, five positive, negative, and neutral words matched for word frequency and length were presented in blue, red, green, or yellow font (McKenna and Sharma 1995). Each of the five words was displayed once per color, producing 20 trials per block. Participants responded to the color of the word with their index, middle, ring, and pinky finger on their dominant hand. Colors were randomly mapped to one of these fingers across participants to avoid systematic effects of finger strength on reaction time (RT). Longer RTs to identify the color of emotional compared to neutral words reflects attentional capture by emotional information, rather than a classic Stroop effect (Algom et al. 2004). This task was used to determine whether any differences in empathic performance across participants emanated from an attentional bias to emotional information. This task was always performed before the patient videos to ensure performance was not influenced by carryover from the more emotional videos. After removing error trials and RTs greater than 3 standard deviations (SD) of each participant's mean (as in Hofelich and Preston 2012), 
attentional capture scores were calculated. Positive and negative bias scores were created for each participant by subtracting median RT to neutral stimuli from the median RT to emotional stimuli. There were no significant differences in capture for positive or negative affect by depression group, $t(60)<1.2, p>.26, \mathrm{~d}<0.29$ or by continuous levels of current depression from the $\mathrm{BDI}, r \mathrm{~s}(58)<.04, p \mathrm{~s}>.75$, replicating our prior work (Cheng et al. 2015). Thus, effects of interest below are unlikely to have derived from attentional bias and are not described further.

\section{Three Main Patient Types and their Qualities}

The 14 videos statistically clustered into three "types" of patients: sad, distraught patients; positive resilient patients; and less emotional reserved patients. While prior studies produced more patient clusters (Preston et al. 2013), this solution still replicated the two main patient types of interest: distraught and resilient. PCA was used to reduce ratings of how the patients appeared to the participants into three other emotion factors - panicked, sad, and softhearted. Average scores for each patient type on these three other emotion factors were created from saved PCA coefficients per extracted factor. PCA also reduced the ratings of how the participant felt while watching the patient into four self emotion factors - personal distress, empathic concern, amusement, and horror. To corroborate the patient groupings into three types and to characterize them, mixed ANOVA compared distraught, resilient, and reserved patients on their other and self factor scores, how sick they seemed, and how much practical and emotional support the seemed to need.

Ratings of the other affect of the patients and of participants' own self responses to them were consistent with prior work (Table 1). Compared to the other two patient types (resilient and reserved), distraught patients appeared to observer participants to be more panicked, sad, sick and in need of emotional and practical support; they also elicited more personal distress, empathic concern, horror, and GSR responsivity (GSR peaks) but lower mean heart rate responses (Table 2). Resilient patients seemed less sad and more softhearted than reserved patients, and they elicited more amusement and were better liked than the other two patient groups. Reserved patients were viewed as the least softhearted and they elicited less empathic concern and more personal distress than resilient patients (post-hoc $p<.001$ ). Ratings of similar life experience, respiration, and mean GSR were similar across patient types (Table 2).

\section{Key Analyses and Results}

\section{Effects of Depression on Patient Affect Categorization}

Participants often sorted patients into more than three groups $(M=4.0$ categories; $S D=1.03$ ), but their self-generated labels revealed broad consensus for at least the three types reported above that we derived from the statistical cluster analysis technique. 36 participants defined a group like distraught (e.g., "seemed extremely sad and depressed" or "very emotionally distressed, very sad"), 12 defined a group like resilient 


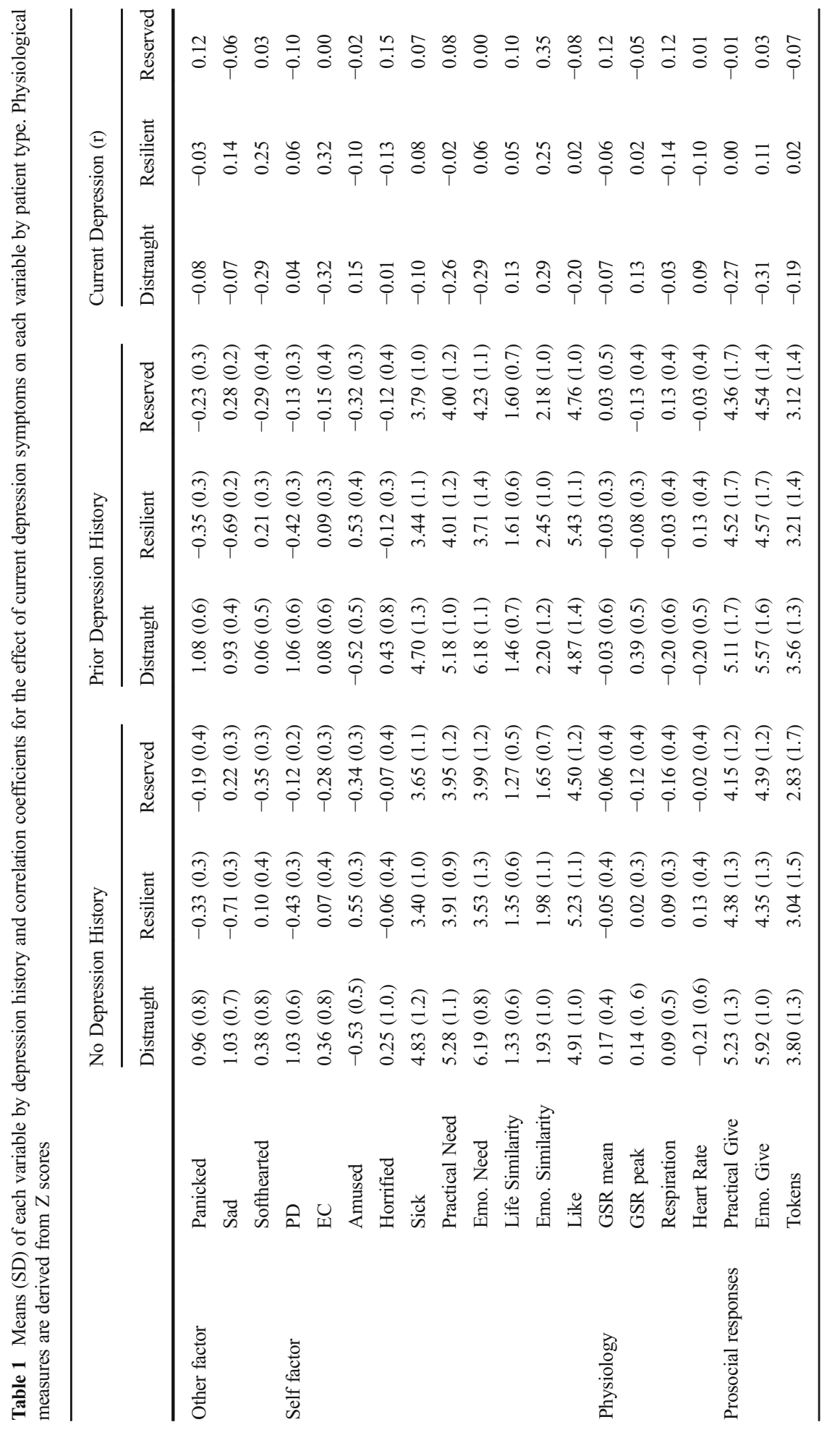


Table $2 F$ values and $\eta^{2}$ for the main effects of patient type, depression history, current depression, as well as interactions between depression experience and patient type (mixed ANOVA with patient type as a withinsubjects factor, past depression experience as a between-subject factor, and BDI score as a covariate). Physiological measures are derived from $\mathrm{Z}$ scores

\begin{tabular}{|c|c|c|c|c|c|c|c|c|c|c|c|}
\hline & & \multicolumn{6}{|c|}{ Main Effects } & \multicolumn{4}{|c|}{$\begin{array}{l}\text { Interactions with } \\
\text { Patient Type }\end{array}$} \\
\hline & & \multicolumn{2}{|c|}{$\begin{array}{l}\text { Patient Type } \\
d f(2,112)\end{array}$} & \multicolumn{2}{|c|}{$\begin{array}{l}\text { Depression } \\
\text { History } \\
d f(1,56)\end{array}$} & \multicolumn{2}{|c|}{$\begin{array}{l}\text { Current } \\
\text { Depression } \\
d f(1,56)\end{array}$} & \multicolumn{2}{|c|}{$\begin{array}{l}\text { Depression } \\
\text { History } \\
d f(2,112)\end{array}$} & \multicolumn{2}{|c|}{$\begin{array}{l}\text { Current } \\
\text { Depression } \\
d f(2,112)\end{array}$} \\
\hline & & $F$ & $\eta^{2}$ & $F$ & $\eta^{2}$ & $F$ & $\eta^{2}$ & $F$ & $\eta^{2}$ & $F$ & $\eta^{2}$ \\
\hline \multirow[t]{3}{*}{ Other factor } & Panicked & $45.20 * *$ & 0.44 & 0.93 & 0.01 & 0.57 & 0.01 & 0.66 & 0.01 & 0.85 & 0.01 \\
\hline & Sad & $95.11 * *$ & 0.63 & 0.01 & 0.000 & 0.19 & 0.001 & 0.38 & 0.002 & 0.40 & 0.003 \\
\hline & Softhearted & $10.26^{* *}$ & 0.15 & 0.59 & 0.01 & $3.48^{+}$ & 0.05 & 0.47 & 0.01 & 2.38 & 0.03 \\
\hline \multirow[t]{10}{*}{ Self factor } & $\mathrm{PD}$ & $51.23 * *$ & 0.48 & 0.03 & 0.000 & 0.05 & 0.000 & 0.001 & 0.000 & 0.15 & 0.001 \\
\hline & $\mathrm{EC}$ & $7.56^{* *}$ & 0.11 & 0.07 & 0.001 & $6.15^{*}$ & 0.09 & 0.71 & 0.01 & $4.22 *$ & 0.06 \\
\hline & Amused & $39.68 * *$ & 0.41 & 0.10 & 0.001 & 1.36 & 0.01 & 0.11 & 0.001 & 0.97 & 0.01 \\
\hline & Horrified & $3.92 *$ & 0.06 & 0.59 & 0.01 & 0.09 & 0.001 & 0.58 & 0.01 & 0.38 & 0.01 \\
\hline & Sick & $42.54 * *$ & 0.42 & 0.30 & 0.001 & 0.01 & 0.000 & 0.19 & 0.002 & 1.59 & 0.02 \\
\hline & $\begin{array}{c}\text { Practical } \\
\text { Need }\end{array}$ & $38.85 * *$ & 0.39 & 1.17 & 0.002 & 0.90 & 0.001 & 0.16 & 0.002 & $3.47 *$ & 0.04 \\
\hline & $\begin{array}{l}\text { Emotional } \\
\text { Need }\end{array}$ & $99.74 * *$ & 0.63 & 2.45 & 0.003 & 1.39 & 0.002 & 0.03 & 0.000 & $2.73^{+}$ & 0.02 \\
\hline & $\begin{array}{l}\text { Life } \\
\text { Similarity }\end{array}$ & 2.33 & 0.04 & $2.98^{+}$ & 0.01 & 0.001 & 0.000 & 2.21 & 0.04 & 1.32 & 0.02 \\
\hline & $\begin{array}{l}\text { Emotional } \\
\text { Similarity }\end{array}$ & 2.20 & 0.04 & 2.51 & 0.02 & $3.02^{+}$ & 0.02 & 1.01 & 0.02 & 0.47 & 0.01 \\
\hline & Like & $4.55^{*}$ & 0.07 & 1.34 & 0.002 & 1.35 & 0.002 & 0.36 & 0.01 & 2.41 & 0.04 \\
\hline \multirow[t]{3}{*}{$\begin{array}{l}\text { Prosocial } \\
\text { responses }\end{array}$} & $\begin{array}{l}\text { Practical } \\
\text { Give }\end{array}$ & $23.60 * *$ & 0.28 & 1.64 & 0.004 & 1.55 & 0.004 & 0.02 & 0.000 & $4.65^{*}$ & 0.06 \\
\hline & $\begin{array}{l}\text { Emotional } \\
\text { Give }\end{array}$ & $41.41 * *$ & 0.40 & 0.52 & 0.001 & 0.47 & 0.001 & 0.12 & 0.001 & $5.99^{*}$ & 0.06 \\
\hline & Tokens & $12.99 * *$ & 0.18 & 0.91 & 0.004 & 0.92 & 0.004 & 1.93 & 0.03 & 2.11 & 0.03 \\
\hline \multirow[t]{4}{*}{ Physiology ${ }^{t}$} & GSR mean & 0.69 & 0.01 & 1.33 & 0.03 & 0.08 & 0.002 & 0.66 & 0.01 & 0.20 & 0.004 \\
\hline & GSR peak & $3.13 *$ & 0.06 & 2.25 & 0.04 & 0.82 & 0.01 & 1.07 & 0.02 & 0.07 & 0.001 \\
\hline & Respiration & 1.13 & 0.02 & $3.21^{+}$ & 0.06 & 0.19 & 0.003 & $6.06^{*}$ & 0.10 & 0.65 & 0.01 \\
\hline & Heart Rate & $3.95^{*}$ & 0.07 & 0.01 & 0.000 & 0.25 & 0.005 & 0.12 & 0.002 & 0.52 & 0.01 \\
\hline
\end{tabular}

$* * p<=.001 ; * p<.05 ;{ }^{+} p<.09$

${ }^{\mathrm{t}} d f$ for physiology tests are $(1,52)$ for main effects of depression history and current depression, $(2,104)$ for rest

(e.g., "worried about their illness, however, were very upbeat and happy"), and 12 defined a reserved group (e.g., "they seemed to have barely any emotion" or "seems more reserved and private than any of the others"). The overall number of groups created did not differ by depression history, $t(57.12)=-1.24, p=.22, \mathrm{~d}=-0.32$, or current depression level, $r(56)=.19, p=.15$. However, supporting the first prediction from the PAM, participants with prior depression experience divided only distraught 
patients into more groups $(M=1.93 ; S D=0.54)$ than those without depression experience $\operatorname{did}(M=1.59 ; S D=0.67), t(57.69)=-2.15, p=.04, \mathrm{~d}=-0.55$. Moreover, a higher current level of depression on the BDI was also associated with the creation of more distraught groups only, $r(55)=.30, p=.03$. The number of divisions for resilient and reserved patients did not differ by depression status, prior history: $t \mathrm{~s}(48.77)<0.95$, $p \mathrm{~s}>.35, \mathrm{~d}<.24$; current level: $r \mathrm{~s}(55)<.20, p \mathrm{~s}>.14$. This enhanced perception of distress in those with depression experience is replicable with statistical clustering of the patients based on the other ratings of patient effect (as we used to find the three overall patient types): If patients are clustered separately for those with and without prior depression, those with prior depression produced a fourth cluster from the distraught group while those without depression produce a fourth cluster from the reserved group.

\section{Effects of Depression on Affective Responses to Patient Types}

In general, participants' prior depression history did not change their perception of the three patient types or the emotions elicited by them, but respiration rates were affected (Table 2). Participants with a prior depression experience had faster respiration rates to distraught $(M=-0.27)$ than reserved patients $(M=.16)$, post-hoc $p=.007$ while participants without depression experience had faster rates to reserved $(M=-0.17)$ than distraught patients $(M=0.13)$, post-hoc $p=.03$. This suggests that a prior depression experience rendered distraught patients more arousing to observers, despite the fact that they were rated similarly.

The continuous degree of current depression (from BDI scores) also did not generally change participants' perception of and response to the three patient types (Table 2). However, higher current depression severity was associated with perceiving distraught patients as needing less practical and emotional support and feeling less empathic concern for them, $\beta=-0.022, t=-2.16, p=.035$; moreover, participants with a higher current depression severity felt more empathic concern for the resilient patients, $\beta=0.014, t=2.75, p=.008$.

\section{Effects of Depression on Prosocial Responses to Patient Types}

Prosocial responses were also assessed with mixed ANOVAs, using ratings of practical and emotional support given as well as actual monetary donations. All participants gave more practical support, emotional support, and tokens to distraught patients than the other two types (Table 2). Prior depression experience was not associated with differences in overall giving or giving to any one patient type. However, mixed regression (with participant as a random factor and patient type as a repeated measure) revealed that only for participants with a prior depression experience did greater similar life experience and emotional experience predict increased practical $(\beta=0.82 ; \beta=0.90)$ and emotional $(\beta=0.65, \beta=0.59)$ support given, interactions for life experience, $F_{\mathrm{S}}(1$, $151.66)>2.91, p \mathrm{~s}<.09$; interactions for emotional experience, $F \mathrm{~s}(1,133.89)>5.40$, $p \mathrm{~s}<.02$. Effects were similar for donated money, with a significant effect for similar life experience, $\beta=1.22 ; F(1,144.74)=5.42, p=.02$, and a non-significant effect in the same direction for similar emotional experience, $\beta=0.65 ; F(1,123.96)=2.39, p=.125$.

Because prior depression history influenced respiration rates across patient types, we performed follow-up tests to determine if these prosocial effects would remain after controlling for respiration by adding it as a covariate to the mixed regression described 
above (average respiration rates by patient type per participant). After adding respiration, the interactions between depression history and similar life experience on emotional support and money donated to patients became non-significant, $F \mathrm{~s}(1$, $140.86)<1.90, p s>.17$ and the effect on practical support given to the patients was reduced, $F(1,134.05)=3.26, p=.07$. However the effect of a similar emotional experience on emotional and practical support given remained significant, $F_{\mathrm{S}}(1$, $131.45)>4.0, p s<.048$.

In contrast to the general enhancement of empathic responding with prior depression experience, participants with increasing current depression severity offered less practical, $\beta=-0.05, t=-2.45, p=.02$, and emotional support, $\beta=-0.05, t=-2.37, p=.02$, to distraught patients only, all other $t \mathrm{~s}<0.53, p \mathrm{~s}>.60$ (Table 2). This effect appears to emanate at least partly from the fact that currently depressed participants perceived distraught patients as needing less practical support (above), as ratings of practical need mediated declining empathy in those with higher current depression symptoms, mediation analysis using multiple step-wise regression, $\mathrm{Z}=-2.05, p=.041$ (Baron and Kenny 1986).

\section{Discussion}

The necessity and importance of shared experience in our empathic ability is complex and still debated. The perception-action model argues that similar past experience is required for empathy because prior personal experiences create more elaborated and embodied mental representations of emotional states and situations, which allow observers to better relate to, model, and simulate others' emotional states and situations. These representations, in turn, enhance empathy, empathic accuracy, and tailored care of targets compared to cases where such similar past experiences are lacking (Preston and de Waal 2002; Preston and Hofelich 2012a). In lay terminology, you can only truly relate to another's experience if you have "walked a mile in their shoes."

Despite the dozens of papers that have found effects of similarity in humans and in non-human animals (summarized in Preston and de Waal 2002), there have been targeted experiments that specifically examined effects of similar life experience on empathy or empathic accuracy that failed to find key effects, calling into question the presumption that similarity is required or even beneficial (e.g., Batson et al. 2005; Hodges et al. 2010). We argue that similarity is not uniformly effective in laboratory experiments because people can be similar at many different levels. The empathic response is only enhanced by similarity at the level of the content of the observer's representation of the state and situation-for example, when the observer and target share knowledge, beliefs, displays, and feelings about the relevant event. In contrast, there should not be an increase in empathic responding when the observer has only had a similar nominal experience, such as an event labeled with the same word (e.g., illness, divorce) or a group affiliation (e.g., gender, school affiliation, political party) that nonetheless produced different subjective experiences in the observer and target.

The current study used a prior experience with depression as a case study for extensive experience with sad, distressed affect. We predicted that having had this experience in the past would allow observers to better perceive distinctions among targets expressing negative affect, which in turn would allow them to be more empathic and helpful toward particularly relatable sad patients. This interactional approach is 
particularly important in the context of depression, because depression is known to manifest itself in multiple, highly divergent ways (e.g., with flat or excessive affect, withdrawing or acting out, sleeping often or too little, losing or gaining weight). As such, one individual who has been depressed may not feel at all similar to another deeply sad person who is not clinically depressed or who is depressed but experienced or displayed the state in another way.

We replicated prior studies from our laboratory using a similar methodology and found that target patients can be classified on the basis of their rated affect, which in turn elicits distinct emotional responses in observers. There are minor differences in the number of patient types or the labeling of the factors depending upon whether the target patients in the videos are grouped statistically or through card-sorting, or separately in each depression group versus across the whole sample. Regardless, some of the patients - all of whom were experiencing serious chronic or terminal illness - can be classified as more sad and distraught, more happy and resilient, and more reserved and showing little affect. In the current study, there were almost no differences across previously depressed, currently depressed, and non-depressed participants in this perception of patients and their response to them, attesting to the robustness of the method. There were only a few differences, which were largely predicted by the model and accord with the two predictions from the introduction, that prior depression would allow participants to perceive more nuanced gradations of sadness, which in turn would enhance empathy and helping for the most similar others. We turn to those results next.

\section{Depression Enhances Representations of Sad Affect}

As predicted, participants with a prior depression experience and with higher current depression severity did perceive greater distinctions among the displays of sadness and distress in the patients, as they grouped only this type of patient into more subgroups on the card-sorting task at the end. This effect was also replicable with statistical clustering techniques. Presumably, this reflects the fact that the extensive experience with intense or prolonged negative affect associated with depression produces richer, more differentiated representations of distress that allows observers to perceive and experience finer gradations of the state in others.

This finding may appear in contrast to prior work that found that people with Major Depressive Disorder (MDD) provide less differentiated descriptions of their own negative emotional experiences than non-depressed individuals (Demiralp et al. 2012). However, these two findings do not necessarily conflict since identifying the emotional states of relatable other individuals after experiencing depression or while currently experiencing some level of depression is different from reporting upon your own feelings during an acute phase of major depression. For example, the apathy, lethargy, and hopelessness associated with depression can blunt the ability or motivation to introspect upon one's own state, to take an interest in its complexity, or to want to describe or discuss it, even when one's internal representations are indeed complex and differentiated. Our study also focused on individuals who had been depressed in the past, some of whom did still feel some depression, but most of whom did not have BDI scores that would quality them as in the midst of a major depressive episode as was the case in the study by Demiralp and colleagues. Moreover, many studies have found negative biases for attributions about the self in depression but not about others 
(e.g., Sweeney et al. 1982; Weary et al. 1987). Thus, depression may leave intact the ability to reflect with precision on external sources of information even if it impairs the ability to reflect upon internal states; in addition, depression may only degrade the complexity of self-report during acute phases of the disease. Further research is warranted on these points.

\section{Prior Depression Enhances Empathy for Similar Others}

Supporting the second hypothesis, even though all participants generally perceived and responded to the hospital patients similarly, previously depressed participants only had a higher respiration rate only while watching the videos of the distraught patients, and they also offered patients more emotional support, practical support and money-but only when they also reported a high level of similarity to the patient in life experience or emotional experience. Thus, presumably, even though many individuals can be said to have experienced depression and a high or sustained level of sadness or distress, there are also bound to be differences in the quality of this experience, such as the feelings it evoked, what precipitated it, and how it is expressed outwardly. These differences produce heterogeneity in the empathic response, even when a target and observer nominally shared a distressing experience, which explains why our own previously depressed participants only felt more empathic and helpful toward patients to whom they felt similar.

This effect of prior depression history is unlikely to have derived from an attentional bias to negative information because previously depressed participants did not uniformly feel more empathic toward distraught patients and they did not have higher attentional capture for positive or negative affect on our separate experimental task (replicating Cheng et al. 2015). However, participants with a prior depression experience did have higher respiration to distraught targets - despite the fact that they did not report feeling more distressed by them. This potentially explains some of the variance in increased prosociality toward similar patients (on life experience), because this effect dropped below significance after accounting for respiration. However, the nearly identical effect of prior depression increasing prosocial responses to patients who were similar on emotional experience remained significant even after adding respiration as a covariate. Thus, prior depression in general does not seem to influence the way patients are perceived, but it may be a more arousing, affective experience for people observing targets in need who remind them of themselves or their own prior struggles in a way that predisposes aid.

\section{Current Depression Impedes Empathy}

In contrast to the largely beneficial effects of prior depression on the response to similar patients, current depression appeared to almost minimize the need of more similar others, as these participants gave less to distraught patients and more to the positive, resilient patients, who could potentially may make them feel better. This latter effect may have captured the alternative "warm glow" motivation to help others that has been previously documented, in which people who are sad or become contagiously sad offer help so that they can feel better (e.g., Andreoni 1995; Manucia et al. 1984). In our study, the greater one's current level of depression, the less the distraught patients 
appeared to need help, the less empathic concern they felt for them, and the more empathic concern they felt for the opposing, happy, resilient patients. Moreover, higher current depression produced lower offers of emotional and practical support to the distraught patients.

Thus, current depression appears to predispose a distancing response toward the distraught patients while favoring positive, resilient patients. This reversal in prosociality occurred despite the fact that participants with higher current depression exhibited a similar level of attentional capture to emotional information in general (negative or positive), a similar physiological response to distraught targets, and equivalent levels of feeling similar, liking the patients, feeling distressed by them, and viewing them as sick or distressed. However, the reduced the perception that distraught patients needed practical support mediated the decreased empathy in follow-up analyses.

A separate study that induced temporary sadness in non-depressed participants did not find decreased giving to distraught patients (Hofelich 2012); this suggests that sadness per se does not necessarily inhibit giving or produce this effect. According to the PAM, such distancing may reflect depressed participants' conflicting goals, which can down-regulate resonance through attentional and cognitive mechanisms (Preston and de Waal 2002). In addition, the negative self-focus associated with disorders like depression (Ingram 1990) may have caused depressed participants to attend to their own feelings and needs, rendering those of others less important, or precluding them from resonating with the distressed patients. It is also possible that feeling depressed reduces your perception of how much help another sad individual needs, because chronically experiencing a similar state may normalize it or render it less unusual or necessitating immediate action. As such, future work should examine the extent to which motivated reasoning (Kunda 1990) or anchoring and adjustment (Epley et al. 2004) account for the minimization of similar others' need that we observed here in depressed participants.

It is also possible that depressed participants are actually more accurate when they infer less need in distraught patients rather than non-depressed participants being more accurate at inferring greater need. We cannot test this with the current data, but depression (e.g., Alloy and Abramson 1979) and negative mood (e.g., Sinclair and Mark 1995) have been associated with increased accuracy in judgments with objective criteria. Moreover, lower ratings of need from those who feel depressed could be consistent with an evolutionary theory that sad mood is an adaptation naturally experienced by those who are trapped in a maladaptive situation and need to regroup to make a change (e.g., Nesse 1990). Future work should replicate this finding with a protocol that has a more objective measure of the target's level of need. We could also potentially explain more variation in the empathic response if we administered a Structured Clinical Interview for Depression (SCID) to participants and targets, so that we can assess the severity and subtype of depression at a more precise level in both populations (e.g., Major Depressive Disorder, Dysthymia, single Depressive Episode).

\section{Concluding Remarks}

The current study suggests that depression has important, diverging consequences for empathy. Important predictions of the PAM have been confirmed, which also resolve much of the confusion about the degree to which similar past experience is required for 
or benefits the empathic response. We confirmed that similar past experience does matter, but is particularly predictive if you assess the content of observers' representations, to be sure that they actually match that of the target. Only when they match at the level of content do they predict prosocial responding. Moreover, even given similar emotional experiences, observers will not empathize if their own current state diverts their attention away from the target or minimizes their need due to conflicting goal states or the requirement to focus upon one's own needs. Future work should extend this knowledge into applied domains, for example by examining the degree to which grouping patients into therapy groups of similar current states enhances versus impedes collective empathy, especially for those in acute phases of the disease. It can be hard to focus on even the most relatable targets when we ourselves are in need.

Acknowledgments This research was supported by a grant from the Rackham Graduate School at the University of Michigan to AHM. The authors thank Jamie Cohen, Kendall Arslanian, Kari Jones, Rebecca Semel, and Eugenia Chen for help with subject testing and data pre-processing.

\section{References}

Algom, D., Chajut, E., \& Lev, S. (2004). A rational look at the Emotional Stroop phenomenon: A generic slowdown, not a Stroop effect. Journal of Experimental Psychology: General, 133(3), 323-338. doi:10. 1037/0096-3445.133.3.323.

Alloy, L. B., \& Abramson, L. Y. (1979). Judgment of contingency in depressed and nondepressed students: Sadder but wiser? Journal of Experimental Psychology: General, 108(4), 441-485.

Andreoni, J. (1995). Warm-glow versus cold-prickle: Effects of positive and negative framing on cooperation in experiments. The Quarterly Journal of Economics, 110(1), 1-21.

Barnett, M. A. (1984). Similarity of experience and empathy in preschoolers. Journal of Genetic Psychology, 145(2), 241-250.

Barnett, M. A., Tetreault, P. A., Esper, J. A., \& Bristow, A. R. (1986). Similarity and empathy: the experience of rape. Journal of Social Psychology, 126, 47-50.

Baron, R. M., \& Kenny, D. A. (1986). The moderator-mediator variable distinction in social psychological research: conceptual, strategic, and statistical considerations. Journal of Personality and Social Psychology, 51(6), 1173-1182.

Batson, C. D., Fultz, J., \& Schoenrade, P. A. (1987). Distress and empathy: two qualitatively distinct vicarious emotions with different motivational consequences. Journal of Personality, 55(1), 19-39.

Batson, C. D., Sympson, S. C., Hindman, J. L., Decruz, P., Todd, R. M., Weeks, J. L., et al. (1996). “I’ve been there, too": Effect on empathy of prior experience with a need. Personality and Social Psychology Bulletin, 22(5), 474 482. doi:10.1177/0146167296225005.

Batson, C. D., Sager, K., Garst, E., Kang, M., Rubchinsky, K., \& Dawson, K. (1997). Is empathy-induced helping due to self-other merging? Journal of Personality and Social Psychology, 73(3), 495-509. doi:10. 1037/0022-3514.73.3.495.

Batson, C. D., Lishner, D. A., Cook, J., \& Sawyer, S. (2005). Similarity and nurturance: Two possible sources of empathy for strangers. Basic and Applied Social Psychology, 27(1), 15-25. doi:10.1207/ s15324834basp2701_2.

Beck, A. T., Steer, R. A., \& Carbin, M. G. (1988). Psychometric properties of the Beck depression Inventory: Twenty-five years of evaluation. Clinical Psychology Review, 8(1), 77-100. doi:10.1016/0272-7358(88) 90050-5.

Blazer, D. G., Kessler, R. C., McGonagle, K. A., \& Swartz, M. S. (1994). The prevalence and distribution of major depression in a national community sample: the national comorbidity survey. The American Journal of Psychiatry, 151(7), 979-986.

Bylsma, L. M., Morris, B. H., \& Rottenberg, J. (2008). A meta-analysis of emotional reactivity in major depressive disorder. Clinical Psychology Review, 28(4), 676-691. doi:10.1016/j.cpr.2007.10.001. 
Cheng, P., Preston, S. D., Jonides, J., Hofelich Mohr, A., Thummala, K., Casement, M., et al. (2015). Evidence against mood-congruent attentional bias in major depressive disorder. Psychiatry Research. doi:10.1016/j. psychres.2015.09.043.

Davis, M. H. (1980). A multidimensional approach to individual differences in empathy. JSAS Catalog of Selected Documents in Psychology, 10, 85.

Davis, M. H. (1983). The effects of dispositional empathy on emotional reactions and helping: A multidimensional approach. Journal of Personality, 51(2), 167-184.

Demiralp, E., Thompson, R., Mata, J., Jaeggi, S., Buschkeuhl, M., Barrett, L. F., et al. (2012). Feeling blue or turquoise? Emotional differentiation in major depressive disorder. Psychological Science, 23(11), 14101416. doi:10.1177/0956797612444903.

Eisenberg, N., \& Fabes, R. A. (1990). Empathy: Conceptualization, measurement, and relation to prosocial behavior. Motivation and Emotion, 14(2), 131-149.

Eisenberg, N., \& Sulik, M. J. (2012). Comment: is self-other overlap the key to understanding empathy? Emotion Review, 4(1), 34-35. doi:10.1177/1754073911421381.

Eisenberg, N., Wentzel, M., \& Harris, J. D. (1998). The role of emotionality and regulation in empathy-related responding. The School Psychology Review, 27(4), 506-521.

Epley, N., Keysar, B., Van Boven, L., \& Gilovich, T. (2004). Perspective taking as egocentric anchoring and adjustment. Journal of Personality and Social Psychology, 87(3), 327-339. doi: 10.1037/0022-3514.87.3.327.

Feshbach, N. D., \& Roe, K. (1968). Empathy in six- and seven-year-olds. Child Development, 39(1), 133145 .

Field, T. (1995). Infants of depressed mothers. Infant Behavior and Development, 18, 1-13. doi:10.1080/ 10673220902899714.

Fritzsche, A., Dahme, B., Gotlib, I. H., Joorman, J., Magnussen, H., Watz, H., et al. (2010). Specificity of cognitive biases in patients with current depression and remitted depression and in patients with asthma. Psychological Medicine, 40(5), 815-826. doi:10.1017/S0033291709990948.Specificity.

Gawronski, I., \& Privette, G. (1997). Empathy and reactive depression. Psychological Reports, 80, 10431049.

Hauser, D. J., Preston, S. D., \& Stansfield, R. B. (2014). Altruism in the wild: when affiliative motives to help positive people overtake empathic motives to help the distressed. Journal of Experimental Psychology. General, 143(3), 1295-1305. doi:10.1037/a0035464.

Hodges, S. D. (2005). Is how much you understand me in your head or mine? In B. F. Malle \& S. D. Hodges (Eds.), Other Minds: How Humans Bridge the Divide between Self and Others (pp. 298-309). New York, NY: Guilford Publications.

Hodges, S. D., Kiel, K. J., Kramer, A. D. I., Veach, D., \& Villanueva, B. R. (2010). Giving birth to empathy: the effects of similar experience on empathic accuracy, empathic concern, and perceived empathy. Personality \& Social Psychology Bulletin, 36(3), 398-409. doi:10.1177/0146167209350326.

Hofelich, A. J. (2012). The role of unique personal representations in understanding and responding to the emotions of others. Doctoral dissertation

Hofelich, A. J., \& Preston, S. D. (2012). The meaning in empathy: Distinguishing conceptual encoding from facial mimicry, trait empathy, and attention to emotion. Cognition \& Emotion, 26(1), 119-128. doi:10. 1080/02699931.2011.559192.

Ingram, R. E. (1990). Self-focused attention in clinical disorders: Review and a conceptual model. Psychological Bulletin, 107(2), 156-176. doi:10.1037/0033-2909.107.2.156.

Jones, N. A., Field, T., \& Davalos, M. (2000). Right frontal EEG asymmetry and lack of empathy in preschool children of depressed mothers. Child Psychiatry and Human Development, 30(3), 189-204.

Joormann, J., \& Gotlib, I. H. (2007). Selective attention to emotional faces following recovery from depression. Journal of Abnormal Psychology, 116(1), 80-85. doi:10.1037/0021-843X.116.1.80.

Krebs, D. (1975). Empathy and altruism. Journal of Personality and Social Psychology, 32(6), 1134-1146.

Kunda, Z. (1990). The case for motivated reasoning. Psychological Bulletin, 108(3), 480-498. doi:10.1037/ 0033-2909.108.3.480.

Lamm, C., Batson, C. D., \& Decety, J. (2007). The neural substrate of human empathy: effects of perspectivetaking and cognitive appraisal. Journal of Cognitive Neuroscience, 19(1), 42-58. doi:10.1162/jocn.2007. 19.1.42.

Manucia, G. K., Baumann, D. J., \& Cialdini, R. B. (1984). Mood influences on helping: Direct effects or side effects? Journal of Personality and Social Psychology, 46(2), 357-364. doi:10.1037/0022-3514.46.2.357.

McKenna, F. P., \& Sharma, D. (1995). Intrusive cognitions: an investigation of the emotional Stroop task. Journal Of Experimental Psychology. Learning Memory And Cognition, 21(6), 1595-1607. doi:10.1037/ 0278-7393.21.6.1595. 
Mischel, W., \& Shoda, Y. (1995). A cognitive-affective system theory of personality: reconceptualizing situations, dispositions, dynamics, and invariance in personality structure. Psychological Review, 102(2), 246-268. doi:10.1037/0033-295X.102.2.246.

Nesse, R. M. (1990). Evolutionary explanations of emotions. Human Nature, 1(3), 261-289. doi:10.1007/ BF02733986.

O’Connor, L. E., Berry, J. W., Weiss, J., \& Gilbert, P. (2002). Guilt, fear, submission, and empathy in depression. Journal of Affective Disorders, 71(1-3), 19-27.

O’Connor, L. E., Berry, J. W., Lewis, T., Mulherin, K., \& Crisostomo, P. S. (2007). Empathy and depression: the moral system on overdrive. In T. F. D. Farrow \& P. W. R. Woodruff (Eds.), Empathy in Mental illness (pp. 49-75). Cambridge University Press: Cambridge.

Paelecke-Habermann, Y., Pohl, J., \& Leplow, B. (2005). Attention and executive functions in remitted major depression patients. Journal of Affective Disorders, 89(1-3), 125-135. doi:10.1016/j.jad.2005.09.006.

Preston, S. D., \& de Waal, F. B. M. (2002). Empathy: Its ultimate and proximate bases. The Behavioral and Brain Sciences, 25(1), 1-71. doi:10.1017/S0140525X02510013.

Preston, S. D., \& Hofelich, A. J. (2012a). The many faces of empathy: Parsing empathic phenomena through a proximate, dynamic-systems view of representing the other in the self. Emotion Review, 4(1), 24-35. doi: 10.1177/1754073911421378.

Preston, S. D., \& Hofelich, A. J. (2012b). Author reply: Understanding Empathy by Modeling Rather than Organizing Its Contents. Emotion Review, 4(1), 38-39. doi:10.1177/1754073911421397.

Preston, S. D., Hofelich, A. J., \& Stansfield, R. B. (2013). The ethology of empathy: a taxonomy of real-world targets of need and their effect on observers. Frontiers in Human Neuroscience, 7(488). doi:10.3389/ fnhum.2013.00488.

Rosekrans, M. A. (1967). Imitation in children as a function of perceived similarity to a social model and vicarious reinforcement. Journal of Personality and Social Psychology, 7(3), 307-315.

Sinclair, R. C., \& Mark, M. M. (1995). The effects of mood state on judgemental accuracy: processing strategy as a mechanism. Cognition \& Emotion, 9(5), 417-438.

Substance Abuse and Mental Health Services Administration. (2006). Results from the 2005 National Survey on Drug Use and Health: National Findings. Rockville, MD: DHHS Publication No. SMA 06-4194.

Sweeney, P. D., Shaeffer, D., \& Golin, S. (1982). Attributions about self and Others in depression. Personality and Social Psychology Bulletin, 8(1), 37-42. doi:10.1177/014616728281006.

Ward, J. H. J. (1963). Hierarchical grouping to Optimize an Objective Function. Journal of the American Statistical Association, 58(301), 236-244.

Weary, G., Elbin, S., \& Hill, M. G. (1987). Attributional and social comparison processes in depression. Journal of Personality and Social Psychology, 52(3), 605-610.

Zahn-Waxler, C., Radke-Yarrow, M., Wagner, E., \& Chapman, M. (1992). Development of concern for others. Developmental Psychology, 28(1), 126-136. doi:10.1037/0012-1649.28.1.126. 